\title{
Conjunctival Metastasis of a Cutaneous Melanoma
}

\author{
Niels J. Brouwer ${ }^{a}$ Marina Marinkovic ${ }^{a} \quad$ Anouk Jochems $^{b}$ Ellen W. Kapiteijn ${ }^{b}$ \\ Sjoerd G. van Duinen ${ }^{c}$ Barbara I. Haeseker ${ }^{d}$ Martine J. Jager ${ }^{a}$ \\ Gregorius P.M. Luyten ${ }^{a}$ \\ Departments of a Ophthalmology, ${ }^{\mathrm{b}}$ Medical Oncology, and ${ }^{\mathrm{c} P a t h o l o g y, ~ L e i d e n ~ U n i v e r s i t y ~ M e d i c a l ~ C e n t r e, ~ a n d ~}$ \\ dDepartment of Ophthalmology, Alrijne Hospital, Leiden, The Netherlands
}

\section{Established Facts}

- Cutaneous melanoma may metastasize to various locations, including the conjunctiva.

- New treatments for metastasized cutaneous melanoma are currently available.

\section{Novel Insights}

- Ophthalmologists should be aware that systemic work-up and a proper medical history are required to differentiate metastases of cutaneous melanoma from primary conjunctival melanoma, thus allowing patients to benefit from the newly introduced treatments.

\section{Keywords}

Melanoma - Metastasis · Conjunctiva - Immunotherapy ·

Treatment

\begin{abstract}
Purpose: To report a patient who presented with a conjunctival tumour as a first sign of distant metastasis of cutaneous melanoma. The patient was treated successfully with BRAF/ MEK-inhibitors and anti-PD-1 antibodies. Methods: Clinical and histopathological examination of the conjunctival lesion. Results: A 74-year-old man was referred to our hospital with a pigmented conjunctival tumour, 5 months after having been diagnosed with cutaneous melanoma on his right
\end{abstract}

\section{KARGER}

(c) 2017 S. Karger AG, Basel

E-Mail karger@karger.com

www.karger.com/oop scapula with loco-regional axillary lymph node metastases. The conjunctival lesion was excised and showed a BRAF V600E mutation. Histopathology showed a melanoma with characteristics suspicious for metastasis, as the lesion did not have a relation with the overlying epithelium. Systemic screening showed multiple distant metastases of the cutaneous melanoma in spleen, liver, and bone. Systemic treatment with the combination of a BRAF-inhibitor (dabrafenib) and MEK-inhibitor (trametinib) was started and followed by a switch to an anti-PD-1 antibody (pembrolizumab). Twenty-two months later, the patient is alive and in good clinical health. Conclusion: Conjunctival metastases of cutaneous melanoma may mimic primary conjunctival melanoma. A good medical history and systemic work-up are required to 
differentiate these diseases. Identification of the proper diagnosis including mutation analysis is crucial, allowing patients to benefit from newly introduced treatment strategies for metastatic cutaneous melanoma.

(c) 2017 S. Karger AG, Basel

\section{Introduction}

Since melanocytes are naturally widespread in the human body, melanoma can develop as a primary malignancy at various locations. Most commonly, it develops as a primary melanoma of the skin. In ophthalmology, melanoma can arise from the uvea and conjunctiva. $\mathrm{Cu}-$ taneous melanoma can spread via the lymph system or by haematogenous dissemination. Metastases are often located in subcutaneous tissue, visceral organs, the brain, and bone, but other - more rare - locations such as the conjunctiva have been reported $[1,2]$.

In recent years, new therapies have been developed for advanced stages of cutaneous melanoma, acting on specific molecular pathways ("targeted therapy") or stimulating the immune system ("immune checkpoint inhibitors") [3]. BRAF-inhibitors (e.g. dabrafenib, vemurafenib) and MEK-inhibitors (e.g. trametinib, cobimetinib) are examples of targeted therapy. The BRAFmutation is frequently present in melanoma, mostly of the non-chronic sun-exposed skin parts [4], and leads to cell proliferation via the activation of the MAPK pathway, in which MEK proteins are involved. Inhibition of BRAF and MEK counteracts the proliferative effect of this pathway. Anti-PD-1 antibodies (e.g. nivolumab, pembrolizumab) are examples of immune checkpoint inhibitors, blocking the inhibitory signal of Programmed Death 1 receptors on $\mathrm{T}$ cells. This results in upregulation of the immune system to attack tumour cells. Recent clinical trials showed an improved survival in selected patients with advanced cutaneous melanoma treated with targeted or immune checkpoint inhibitor therapy [5].

Clinically, it can be difficult to differentiate primary from secondary malignant melanocytic lesions. This discrimination is very relevant for further treatment, as patients with metastatic cutaneous melanoma might benefit from the aforementioned treatments, which would not be applied to a localized conjunctival melanoma. We describe a patient with a pigmented conjunctival tumour, which turned out to be the first presentation of distant metastasis of a cutaneous melanoma, and who was successfully treated with systemic therapy.
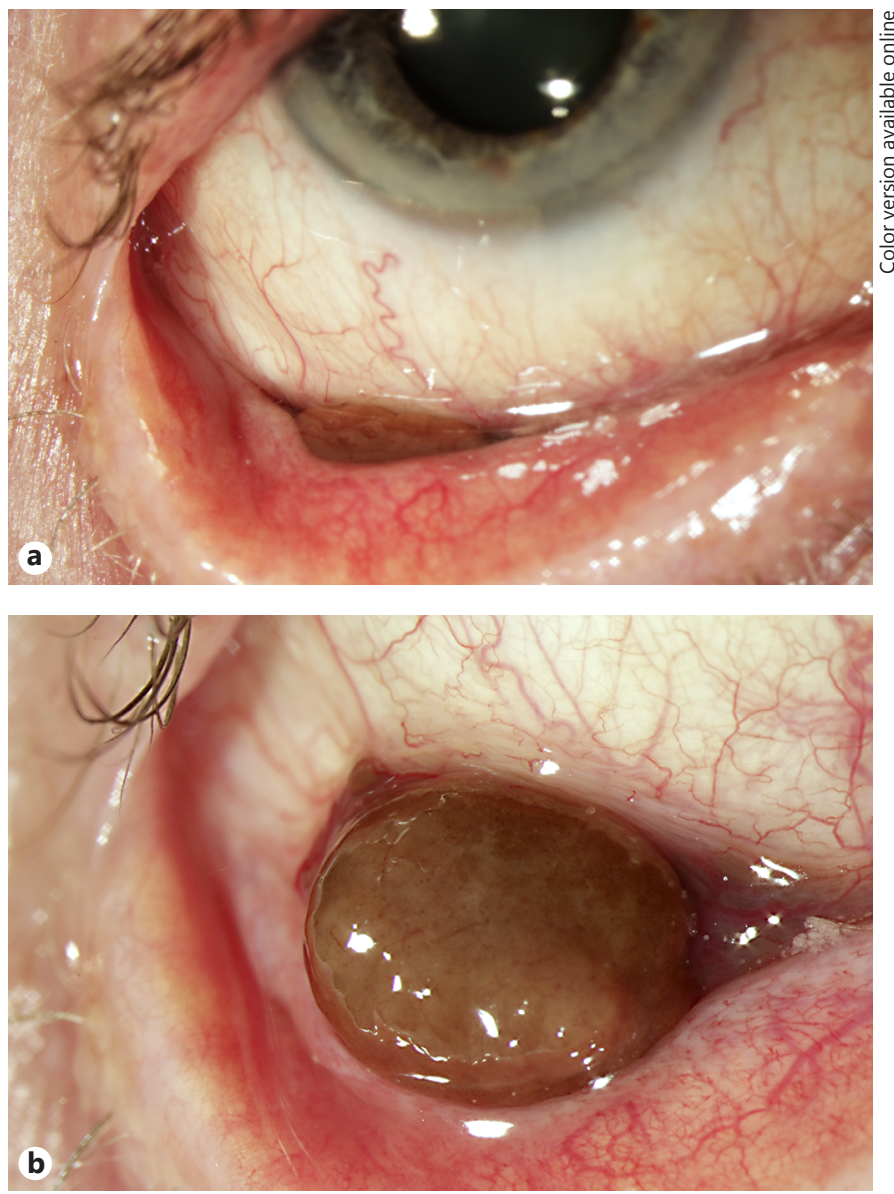

Fig. 1. Slit-lamp photography at presentation. a A pigmented lesion is located in the inferior fornix of the right eye. $\mathbf{b}$ On closer examination, a well-circumscribed nodular lesion is seen with a diameter of $5.0 \mathrm{~mm}$.

\section{Case Report}

A 74-year-old white male was diagnosed in 2015 with a cutaneous melanoma on the right scapular region of the back. The lesion (Breslow thickness $8 \mathrm{~mm}$ ) was completely excised and demonstrated a BRAF V600E mutation. PET-CT screening for metastases revealed suspicious nodes in the ipsilateral axilla, but no other systemic lesions. A lymph node dissection was performed, with 3 out of 13 positive lymph nodes. Postoperative radiation therapy (20 fractions of $2.4 \mathrm{~Gy}$ ) was administered to the axillar region. According to the 7th edition of the AJCC staging manual, the melanoma was classified as a T4aN2bM0 tumour, stage III B.

Five months after the diagnosis, a pigmented tumour was observed in the inferior fornix of the right eye (Fig. 1). The lesion had a distinct border, and no other conjunctival pigmentation was seen. The lesion was excised and histopathology showed a melanoma, positive for the BRAF V600E mutation. The tumour was located in the subepithelial stroma without a component of primary acquired melanosis (PAM) in the overlying epithelium, and therefore a metastasis was suspected of the previously diagnosed 

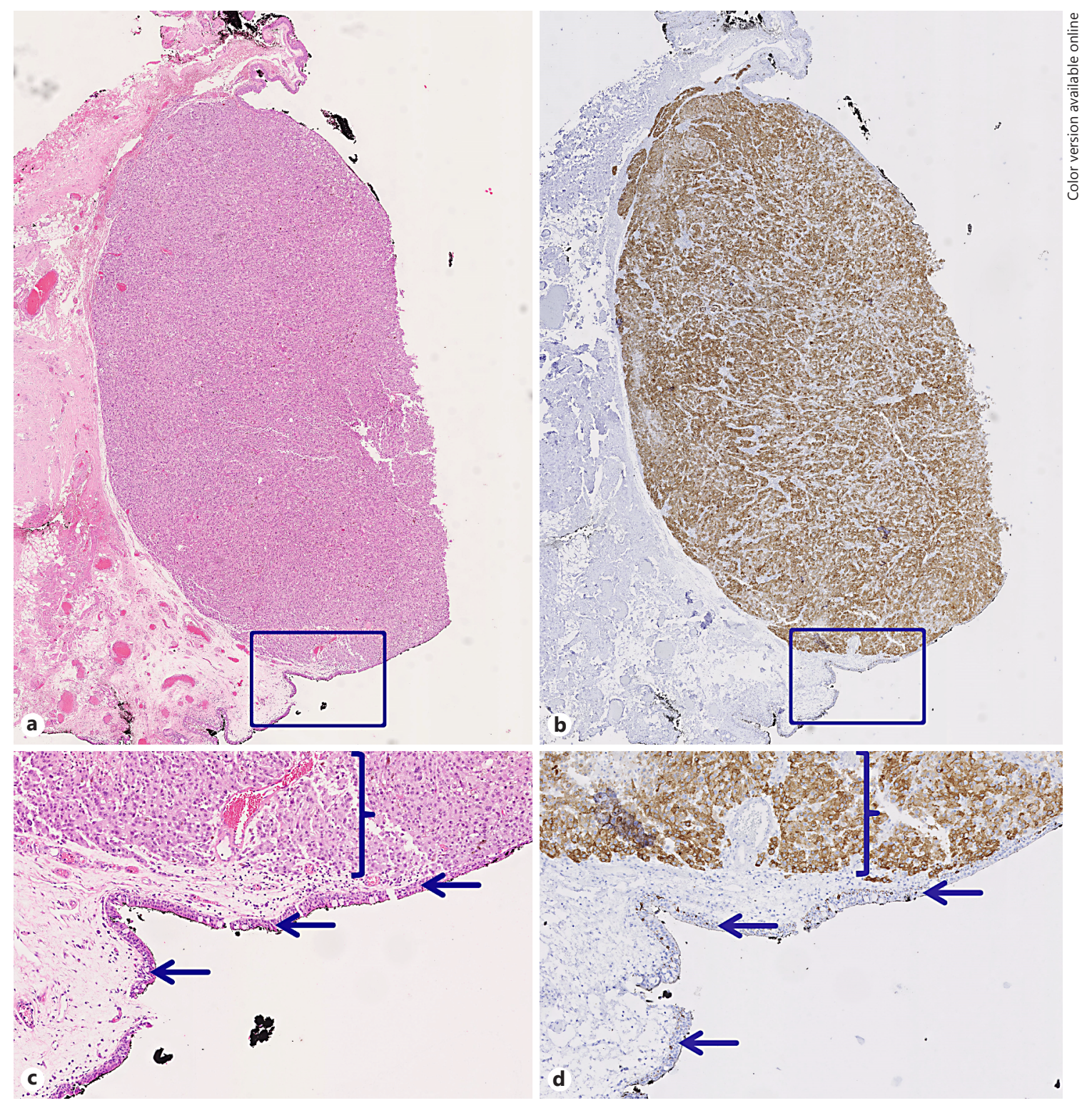

Fig. 2. Histopathology. a Overview of the pigmented lesion, revealing a nodular tumour in the forniceal conjunctiva. The area within the box is presented at a higher magnification in c (HE staining, original magnification $5 \times$ ). b The nodule stains positive for MelanA, indicating a melanocytic origin of the cells, suggestive for melanoma. The area within the box is presented at a higher magnification in $\mathbf{d}$ (Melan-A staining, original magnification $5 \times$ ). $\mathbf{c}$ The tu-

cutaneous melanoma (Fig. 2). The primary melanoma showed an epithelioid cell type with similarities to the suspected metastasis in cell size, nuclear size, and cellular configuration (Fig. 3).

A PET-CT scan was repeated and other metastases were subsequently identified in the liver, spleen, and various bones. Treatment with a BRAF-inhibitor was started (dabrafenib $100 \mathrm{mg}$, twice daily) for 4 months, followed by a combined treatment with a MEK-inhibitor (trametinib $2 \mathrm{mg}$, once daily) for another 2 months. mour cells (bracket) are located in the stroma without relation to the conjunctival epithelium (arrows). (HE staining, original magnification $40 \times$ ). d The positively staining melanocytes of the tumour (bracket) are clearly separated from the epithelium (arrows), and no intra-epithelial growth of primary acquired melanosis is present. Together, this suggests a non-primary (metastatic) origin of the lesion (Melan-A staining, original magnification $40 \times$ ).

Due to a mixed response, the treatment regimen was switched to intravenous injections with the anti PD-1 antibody pembrolizumab (200 mg) with 3-week intervals.

Currently, 22 months after the diagnosis of metastatic cutaneous melanoma, the patient is still alive and in good health. No local recurrences or new conjunctival lesions have been observed, and the distant metastases regressed. During treatment with pembrolizumab, a mild skin rash developed for which topical corticoste- 

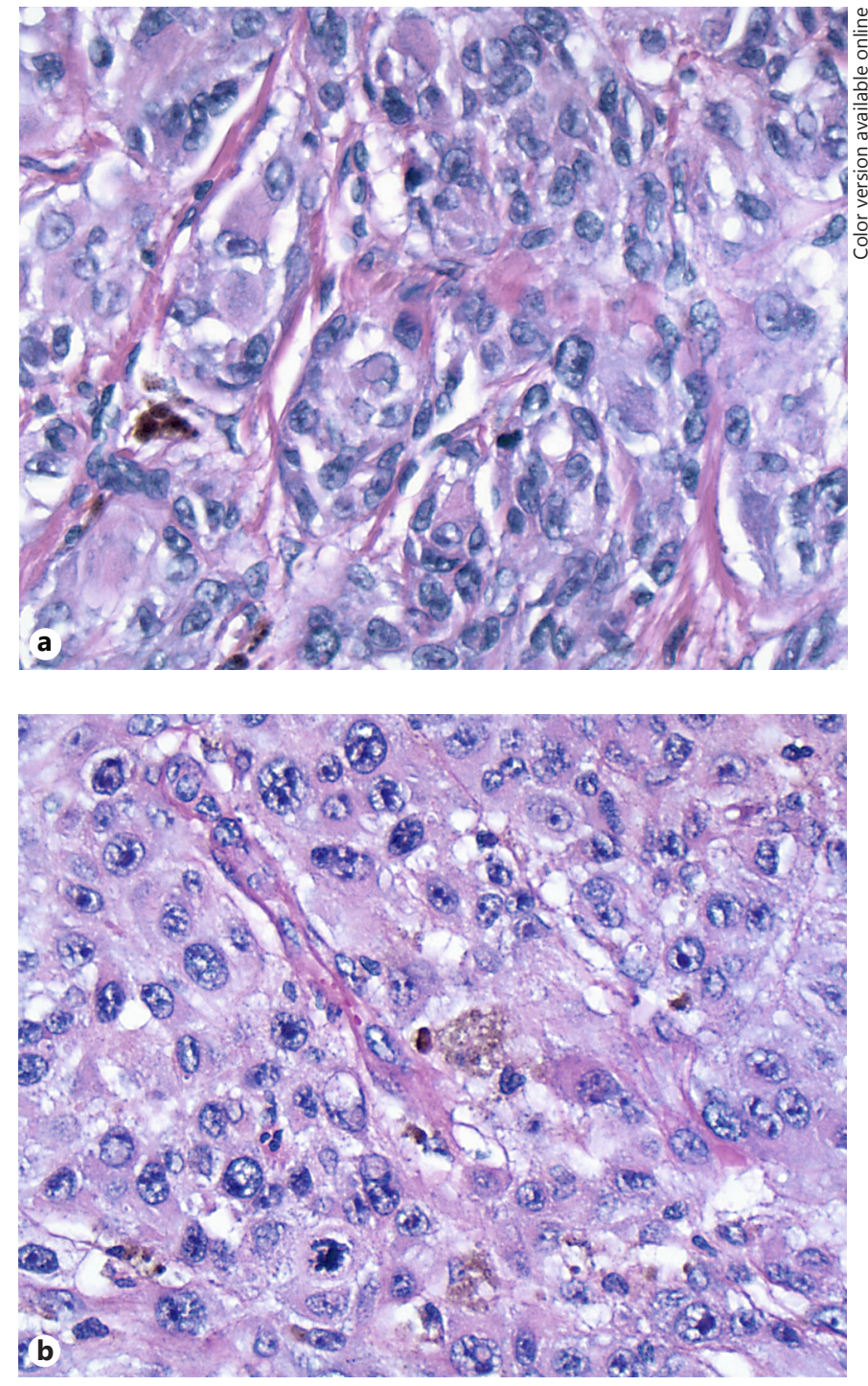

Fig. 3. Cell type of the primary and metastatic lesion. The primary cutaneous melanoma (a) shows an epithelioid cell type, with similarities in cell size, nuclear size, and cellular configuration to the conjunctival metastasis (b). (HE staining, original magnification $40 \times)$.

roids were prescribed, but no other adverse events of the immunotherapy have been noticed. Treatment with pembrolizumab will be continued till disease progression or unacceptable toxicity, for at most another 8 months to a total of 24 months.

\section{Discussion}

Melanomas can develop in the conjunctiva both as a primary or secondary lesion. As a primary tumour, conjunctival melanomas originate from the melanocytes in the basal layer of the conjunctiva. With an incidence of up to $0.8 /$ million in Caucasians, it is rare [6]. Conjunctival melanomas can develop de novo, from a nevus, but most frequently they develop from PAM [7]. The treatment of primary conjunctival melanomas consists generally of local excision with adjuvant treatment of topical chemotherapy, cryotherapy, or brachytherapy [8].

Secondary conjunctival melanomas may result from direct extension or distant metastasis of cutaneous or uveal melanomas $[9,10]$. An overview of 19 conjunctival metastases of cutaneous melanomas showed a poor survival, ranging from $<1$ to 16 months [11]. However, it has to be noted that survival data in 5 out of 19 reported cases were absent $[2,10,12-20]$.

Based on clinical appearance, the pigmented conjunctival lesion of our patient could not be classified as a primary or secondary lesion. Histologic examination showed that no PAM was present in the conjunctiva, and the tumour was located entirely in the subepithelial stroma, which suggested a diagnosis of cutaneous melanoma metastasis. When systemic metastasis screening was repeated, it revealed metastases to other organs. Together with the history of cutaneous melanoma, we suspected the conjunctival lesion to be a distant metastasis as well.

Both the primary cutaneous melanoma and the conjunctival metastasis showed the same BRAF V600E mutation. This similarity, however, should not be seen as a proof of shared origin, since the BRAF mutation may occur in primary conjunctival melanoma as well [21]. This is illustrated by the fact that conjunctival melanomas share a genetic similarity with cutaneous melanomas, rather than with uveal melanomas: BRAF and NRAS mutations that are common in cutaneous melanomas are also seen in conjunctival melanomas, but they are extremely rare in uveal melanomas [22, 23]. GNAQ and GNA1 1 mutations are seen in uveal melanomas, but they have not been identified in conjunctival melanomas [24]. Following these genetic characteristics, some patients with localized and metastatic conjunctival melanomas were treated with BRAF inhibitors $[25,26]$, while other treatments are required for metastatic melanomas of the uvea. Since both melanomas of the skin and of the uvea can metastasize to the conjunctiva, determination of the origin of a metastasis can be very relevant for treatment selection.

In conclusion, a conjunctival metastasis of cutaneous melanoma may mimic primary conjunctival melanoma. In our case, following the discovery of disseminated disease, the patient was treated successfully with new sys- 
temic therapy. Clinicians should always be aware of the possibility of metastasis of cutaneous melanoma to the eye, indicating the importance of a proper medical history and systemic work-up. Newly introduced treatments for metastasized cutaneous melanoma might benefit these patients.

\section{Statement of Ethics} paper.

The patient gave informed consent for the publication of this

\section{Disclosure Statement}

None of the authors has a conflict of interest to disclose.

\section{References}

1 Leiter U, Meier F, Schittek B, Garbe C: The natural course of cutaneous melanoma. J Surg Oncol 2004;86:172-178.

2 Kiratli H, Shields CL, Shields JA, DePotter P: Metastatic tumours to the conjunctiva: report of 10 cases. Br J Ophthalmol 1996;80:5-8.

3 Michielin O, Hoeller C: Gaining momentum: new options and opportunities for the treatment of advanced melanoma. Cancer Treat Rev 2015;41:660-670.

4 Kim SY, Kim SN, Hahn HJ, Lee YW, Choe YB, Ahn KJ: Metaanalysis of BRAF mutations and clinicopathologic characteristics in primary melanoma. J Am Acad Dermatol 2015; 72:1036-1046 e1032.

5 Kee D, McArthur G: Immunotherapy of melanoma. Eur J Surg Oncol 2017;43:594-603.

6 Tuomaala S, Eskelin S, Tarkkanen A, Kivela T: Population-based assessment of clinical characteristics predicting outcome of conjunctival melanoma in whites. Invest Ophthalmol Vis Sci 2002;43:3399-3408.

7 Shields CL, Markowitz JS, Belinsky I, Schwartzstein H, George NS, Lally SE, Mashayekhi A, Shields JA: Conjunctival melanoma: outcomes based on tumor origin in 382 consecutive cases. Ophthalmology 2011;118: 389-395.e381-e382.

8 Wong JR, Nanji AA, Galor A, Karp CL: Management of conjunctival malignant melanoma: a review and update. Expert Rev Ophthalmol 2014;9:185-204.

9 Blumenthal EZ, Garzozi H, Bahir J, Pe'er J: Multiple conjunctival metastases as the initial sign of metastatic uveal melanoma. Am J Ophthalmol 1997;124:549-550.
10 Dhar-Munshi S, Ameen M, Wilson RS: Simultaneous metastases of cutaneous malignant melanoma to conjunctiva and choroid. Br J Ophthalmol 2000;84:930-931.

11 Carey AR, Espana EM, Margo CE: Conjunctival metastasis from a regional cutaneous melanoma. Can J Ophthalmol 2014;49:e97e99.

12 Jakobiec FA, Buckman G, Zimmerman LE, La Piana FG, Levine MR, Ferry AP, Crawford JB: Metastatic melanoma within and to the conjunctiva. Ophthalmology 1989;96:999-1005.

13 Kwapiszeski BR, Savitt ML: Conjunctival metastasis from a cutaneous melanoma as the initial sign of dissemination. Am J Ophthalmol 1997;123:266-268.

14 Shields JA, Shields CL, Eagle RC Jr, Raber IM: Conjunctival metastasis as initial sign of disseminated cutaneous melanoma. Ophthalmology 2004;111:1933-1934.

15 Shields JA, Eagle RC Jr, Gausas RE, Bundy WE 3rd, Shields CL: Retrograde metastasis of cutaneous melanoma to conjunctival lymphatics. Arch Ophthalmol 2009;127:12221224.

16 Strempel I: Metastatic conjunctival tumor originating in a primary cutaneous malignant melanoma [author's transl] (article in German). Ophthalmologica 1982;185:52-57.

17 Ziakas NG, Eke T, Kendall CH, Goulstine DB: Metastatic cutaneous melanoma to the conjunctiva in an Afro-Caribbean patient. Eye (Lond) 2000;14:667-668.

18 Font RL, Naumann G, Zimmerman LE: Primary malignant melanoma of the skin metastatic to the eye and orbit. Report of ten cases and review of the literature. Am J Ophthalmol 1967;63:738-754.
19 Gunther I: Ocular metastases in malignant melanoma of the skin [author's transl] (article in German). Klin Monbl Augenheilkd 1973; 162:821-823.

20 Filik L, Oskay T, Ozyilkan O: Conjunctival involvement in a patient with cutaneous malignant melanoma. Onkologie 2002;25:64-65.

21 Larsen AC, Dahl C, Dahmcke CM, LadeKeller J, Siersma VD, Toft PB, Coupland SE, Prause JU, Guldberg P, Heegaard S: BRAF mutations in conjunctival melanoma: investigation of incidence, clinicopathological features, prognosis and paired premalignant lesions. Acta Ophthalmol 2016;94:463-470.

22 Spendlove HE, Damato BE, Humphreys J, Barker KT, Hiscott PS, Houlston RS: BRAF mutations are detectable in conjunctival but not uveal melanomas. Melanoma Res 2004; 14:449-452.

23 Griewank KG, Westekemper H, Murali R, et al: Conjunctival melanomas harbor BRAF and NRAS mutations and copy number changes similar to cutaneous and mucosal melanomas. Clin Cancer Res 2013;19:31433152.

24 Van Raamsdonk CD, Griewank KG, Crosby $\mathrm{MB}$, et al: Mutations in GNA11 in uveal melanoma. N Engl J Med 2010;363:2191-2199.

25 Maleka A, Astrom G, Bystrom P, Ullenhag GJ: A case report of a patient with metastatic ocular melanoma who experienced a response to treatment with the BRAF inhibitor vemurafenib. BMC Cancer 2016;16:634.

26 Pahlitzsch M, Bertelmann E, Mai C: Conjunctival melanoma and BRAF inhibitor therapy. J Clin Exp Ophthalmol 2014;5:322. 